\title{
LIFEMUSIC: REFLECTION OF LIFE MEMORIES BY DATA SONIFICATION
}

\author{
Ridwan A. Khan ${ }^{1}$, Ram K. Avvari ${ }^{1}$, Katherine Wiykovics ${ }^{1}$, Pooja Ranay ${ }^{2}$, and Myounghoon Jeon ${ }^{1}$ \\ ${ }^{1}$ Computer Science, \\ Michigan Technological University, \\ Houghton, MI 49931, USA \\ \{ridwank, javvari, krwiykovi, mjeon\}@mtu.edu \\ ${ }^{2}$ Electrical and Computer Engineering, \\ Michigan Technological University, \\ Houghton, MI 49931, USA \\ pranay@mtu.edu
}

\section{ABSTRACT}

Memorable life events are important to form the present selfimage. Looking back on these memories provides an opportunity to ruminate meaning of life and envision future. Integrating the life-log concept and auditory graphs, we have implemented a mobile application, "LifeMusic", which helps people reflect their memories by listening to their life event sonifcation that is synchronous to these memories. Reflecting the life events through LifeMusic can relieve users of the present and have them journey to the past moments and thus, they can keep balance of emotions in the present life. In the current paper, we describe the implementation and workflow of LifeMusic and briefly discuss focus group results, improvements, and future works.

\section{INTRODUCTION}

Past events are essential part to form the present self. On one hand, remembering these events can make some people nostalgic. On the other hand, people may evaluate their lives through reflecting past events. To help this self-reflection process, life-logging research and products have been introduced. How can we facilitate this reflection process to be more effective rather than just piling up the data? What if we sonify our life events and listen to them as a music piece? Given that sonification can provide not only cognitive information, but also strong emotional information [1], it might be a plausible approach. Our research started to address these questions. Past memories can be either pleasant or unpleasant and thus, we are able to plot the life events on the Cartesian coordinate with the mapping of the $\mathrm{X}$ axis as time and $\mathrm{Y}$ axis as emotions (e.g., positive-negative valence). Leveraging the life-logging concept and auditory graphs, we have developed an Android app, "LifeMusic", to help people reflect their memories more deeply and remember precious life moments more vividly. People are not able to change the past, but they may be able to connect it to the present and better realize the value of their present lives.

\section{LIFE-LOGGING}

Life-logging is not a new concept. Researchers have tried to trace people's geographical positions using GPS, WiFi [e.g., 2], or wearable camera [3] to support their memory about personal life events. Attempts such as LifeLog [4] or Microsoft MyLifeBits came out with a focus on life-long

\section{(c) (i) (5)}

Attribution - Non Commercial 4.0 International License.

The full terms of the License are available at

http://creativecommons.org/licenses/by-nc/4.0/ information capture. This type of applications concentrated on events, states, and relationships of an individual and aggregated them as raw data into the person's timeline. Some of them pursued the role of growth memories in the intentional cultivation of good life [5]. Another study with older adults [6] focused on remembering what life events (both positive and negative) shaped their lives. From this study, it seems that negative events are less likely to have shaped peoples' lives than positively scripted events, such as marriage, raising kids, etc. Overall, most research has represented the data visually, whereas there has been little research on representing the data auditorily. Based on this background, we posit the hypothesis that providing auditory representations of one's life events (in addition to visuals) will facilitate this reflection process more effectively.

\section{AUDITORY GRAPHS}

The ICAD community has a long history of auditory graph research [e.g., 7-10]. For example, Davison and Walker [10] tried to make a software standard for auditory graphs, "Sonification Sandbox". Developed in Java, it provides a cross-platform tool for auditory display researchers. The Sonification Sandbox converts tabular information into a descriptive auditory graph with various sound profiles, including pitch, timbre, polarity, pan, and volume. It also provides graph contexts, such as tick marks and labels. Given that a graph itself is a complex construct, an auditory graph can be more easily presented to the listeners with this type of context. Smith and Walker [8] empirically showed that an addition of the context to auditory graphs better represented the data than without the context. Nees and Walker [9] also identified additional essential considerations of the auditory graph characteristics: data, mappings, scaling, polarities, context, temporal characteristics of auditory graph stimuli, and multiple data series, all of which we can further consider for our app.

Representing one's life events with an auditory graph has some advantages over just visual representation: (1) Given that life has a time dimension, an auditory modality fits to displaying it effectively; (2) Since sound and music are deeply related to emotions, the sonification of life will promote people's affective contemplation process of their lives; (3) We aim to develop a smartphone app and so sound is an appropriate channel to compensate for its small display. Integrating these ideas, we have developed our application, LifeMusic, which records users' life events and their mood, and represent those events with visual and auditory graphs according to different memory nodes. 


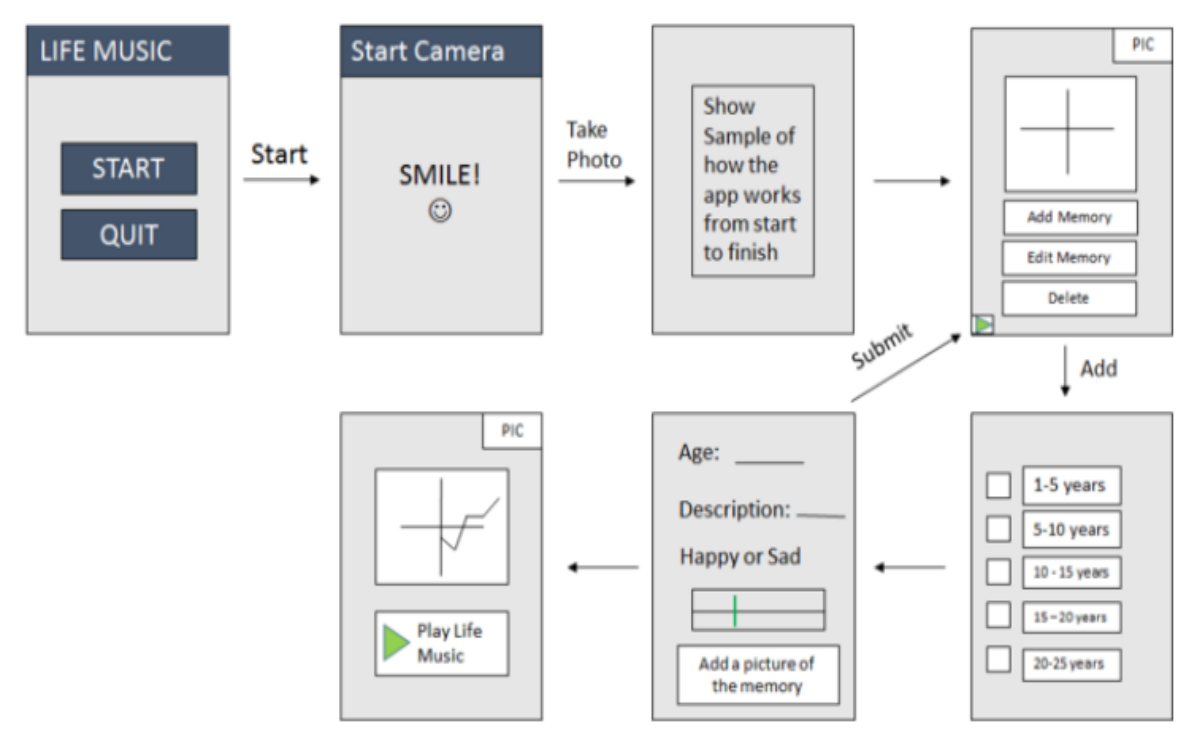

Figure 1. Proposed Model of the App Flow

\section{LIFE MUSIC APP}

LifeMusic application was developed as a smartphone app based on Android OS so that a large number of population could get access to this application and use it on the go. The application has a simple design which starts with a welcome screen (Figures 1-2). Pressing the start button in the welcome screen leads users to the picture capture screen. Users can take a photo or select one from their photo gallery, which is connected to their memory node. After the short demo video of how to use the app, users can see the core part of the application - the plot screen where they can plot their memories as nodes in a visual graph. In this screen, there is an "Add memory" button, which leads to a form screen. In the form screen, users can write about the specific memory. Users can give a title of the memory and write some description and time of the memory. This writing process can induce a specific emotional state linked to their memory [11]. Then, users rate this memory on a sad-happy scale between one to ten where one being absolutely sad and ten being absolutely happy. After saving the data in the form, users are redirected to the plot screen where the memory is added to the graph as a node. Again, in this way, users can add several memory nodes in the graph. In the graph, the $\mathrm{X}$ axis represents the time or age when the memory event happened and the $\mathrm{Y}$ axis represents the sad-happy scale.

We have used a third party API, GraphView [12] to show the graph. There is a button, "Play life music," in the plot screen. When users press this button, a sonification piece is produced and played according to the plotted nodes of the graph. Initially, we have three types of different piano sounds for playing memories: one for happy, one for neutral, and the last one for sad memory events. The sound for each emotional node lasts relatively long (around 7-10 seconds) to facilitate user contemplation and reflection. For our working prototype, we used Android SDK's multimedia framework (MediaPlayer API) to play the sound.

\section{PRELIMINARY EVALUATION}

To receive initial user feedback, we demonstrated our application to eight (4 female) graduate student participants in a single focus group. Their age was between 21 years to 30 years. They have different nationality, including India, China, USA, and Iran. First, the application was demonstrated to the participants and then, each of them experienced the application one by one. After getting used to the application, they could easily use the application to log their life events. Next, they provided their feedback on LifeMusic app. Our participants understood the concept of our app and how it worked very well. Some participants wanted to have more options of emotions (e.g., circumplex model [13]) rather than just happiness and sadness. Participants also suggested that the timeline of a memory can be more specific (e.g., time of the day, week, month, etc.) rather than just a year with a zooming function. Another suggestion was to make the music more rhythmic and varied. Overall, participants were excited about the use of our application and provided considerable ideas and improvements.

\section{IMPROVED SONIFICATION CONCEPTS}

Based on the comments and feedback from the focus group, we are improving our app in a number of aspects. Here, we focus on the description of the sonification part. First iteration is to include a melody contour of the familiar music pieces [e.g., 14]. For example, we can have users mark their events as four seasons: spring, summer, fall, and winter as people often use an analogy of four seasons to describe their lives. Then, we can play each season's theme of Antonio Vivaldi's "Four Seasons" for each life event. The second iteration could be to sonify the life events just as auditory graphs do with more granularity (e.g., using the Sonification Sandbox or other sound engine such as JFugue in Java) beyond just positive and negative valence. We can also include many other variables. For example, we will have users' input on the strength and effect size of each life event 


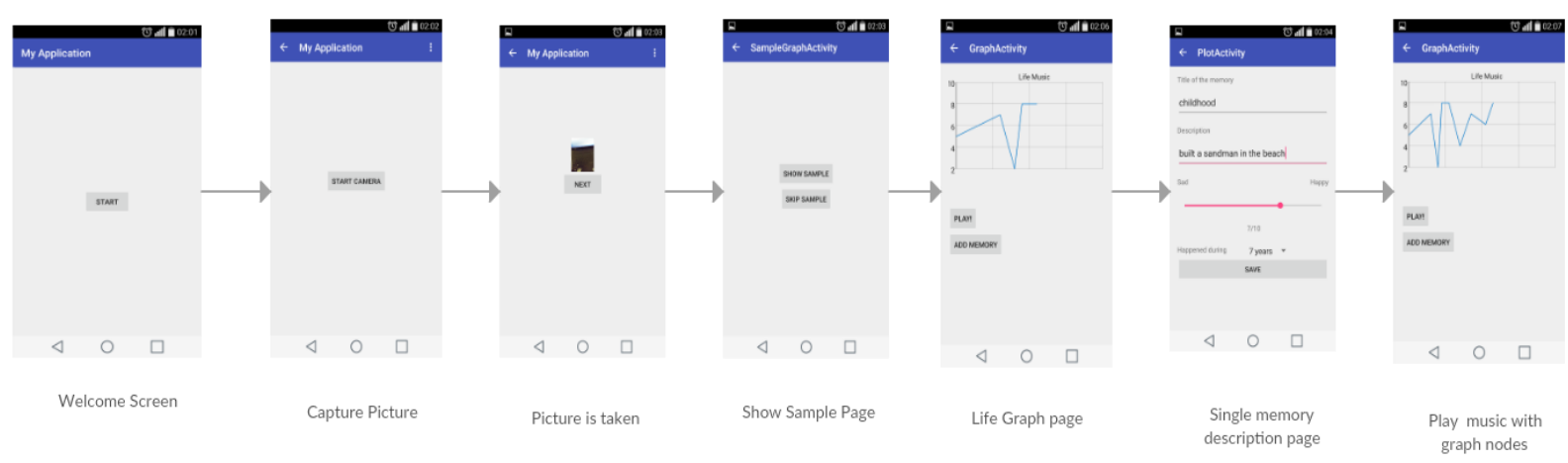

Figure 2. Screen Capture of the Actual LifeMusic App

and those data could be mapped onto musical variables, such as volume or duration of the sound. Depending on people's conceptualization of their life events, the same melody contour can vary and play a different genre of music (e.g., Rock \& Roll vs. Classic vs. Jazz) or different cultural music.

\section{CONCLUSION \& FUTURE WORKS}

LifeMusic is a small step towards a novel application with many possibilities. The aim of this application is to help people trigger their past nostalgic memories and reflect them more effectively. It allows users to gain a new perspective of their past and plan their future. Both happy and sad moments of the past constitute the "music of life". The current application can be improved by adding more subtle emotions rather than the simple valence dimensions. Another extension for this application is to create it for multiple users so that one user can compare and understand his or her own graph with others' by orchestrating them together. Of course, we will also use different visual representations of the graph and improve the sound generation by making it more accessible and customizable. Overall, the project is still a work-inprogress, but we are able to demonstrate it in the conference and hope to get some feedback from the ICAD community. We hope that a simple life graph system like LifeMusic can be a little reminder for people that life is short and we should try to make wonderful memories everyday regardless of being happy or sad.

\section{ACKNOWLEDGMENT}

The authors would like to thank all the students who came and spent their precious time to view and provide feedback about the application.

\section{REFERENCES}

[1] M. Jeon, "Two or three things you need to know about AUI design or designers," in Proc. of Int. Conf. (ICAD2010), Washington, D.C., June, 2010.

[2] J. Rekimoto, T. Miyaki, and T. Ishizawa, "LifeTag: WiFi-based continuous location logging for life pattern analysis.” In LoCA (Vol. 2007, pp. 35-49).
[3] A. J. Sellen, A. Fogg, M. Aitken, S. Hodges, C. Rother, and K. Wood, "Do life-logging technologies support memory for the past?: an experimental study using sensecam," in Proc. (CHI2007) (pp. 81-90). ACM Press. 2007

[4] K. O'Hara, M. M. Tuffield, and N. Shadbolt, "Lifelogging: Privacy and empowerment with memories for life", Identity in the Information Society, vol. 1, no. 1, pp. 155-172, 2009.

[5] J. J. Bauer, D. P. McAdams, and A. R. Sakaeda, "Interpreting the good life: Growth memories in the lives of mature, happy people", Journal of Personality and Social Psychology, pp. 203-217, 2005.

[6] D. Bertsen, D. C. Rubin, and I. C. Siegler, "Two versions of life: Emotionally negative and positive life events have different roles in the organization of life story and identity," Emotion, vol. 11, no. 5, pp. 11901201, 2011.

[7] M. L. Brown, and S. A. Brewster, "Drawing by ear:interpreting sonified line graphs," in Proc. of Int. Conf. (ICAD2003), 2003.

[8] D. R. Smith and B. N. Walker, "Tick-marks, axes, and labels: The effects of adding context to auditory graphs", in Proc. of Int. Conf. (ICAD 2002), 2002.

[9] M. A. Nees, and B. N. Walker, "Listener, task, and auditory graph: Toward a conceptual model of auditory graph comprehension", in Proc. of Int. Conf. (ICAD 2007), 2007.

[10] B. Davison, B. N. Walker, "Sonification Sandbox overhaul: Software standard for auditory graphs," in Proc. of Int. Conf. (ICAD 2007), Montreal, Canada, 2629 June. pp. 509-512, 2007.

[11] G. V. Bodenhausen, L. A. Sheppard, and G.P. Kramer, "Negative affect and social judgment: The differential impact of anger and sadness," European Journal of Social Psychology, vol. 24, pp. 45-62.

[12] http://www.android-graphview.org

[13] J. A. Russel, "A circumplex model of affect," Journal of Personality and Social Psychology, vol. 39, no. 6, pp. 1161-1178, 1980.

[14] B. N. Walker, J. Kim, and A. Pendse, "Musical soundscapes for an accessible aquarium: Bringing dynamic exhibits to the visually impaired," in Proc. of Int. (ICMC 2007), Copenhagen, Denmark, August 2730, 2007. 